\title{
Robust Image-Based Estimation of Cardiac Tissue Parameters and Their Uncertainty from Noisy Data
}

\author{
Dominik Neumann ${ }^{1,2}$, Tommaso Mansi ${ }^{1}$, Bogdan Georgescu ${ }^{1}$, Ali Kamen ${ }^{1}$, \\ Elham Kayvanpour ${ }^{3}$, Ali $\mathrm{Amr}^{3}$, Farbod Sedaghat-Hamedani ${ }^{3}$, Jan $\mathrm{Haas}^{3}$, \\ Hugo Katus ${ }^{3}$, Benjamin Meder ${ }^{3}$, Joachim Hornegger ${ }^{2}$, and Dorin Comaniciu ${ }^{1}$ \\ 1 Imaging and Computer Vision, Siemens Corporate Technology, Princeton, NJ, USA \\ 2 Pattern Recognition Lab, FAU Erlangen-Nürnberg, Germany \\ 3 Department of Internal Medicine III, University Hospital Heidelberg, Germany
}

\begin{abstract}
Clinical applications of computational cardiac models require precise personalization, i.e. fitting model parameters to capture patient's physiology. However, due to parameter non-identifiability, limited data, uncertainty in the clinical measurements, and modeling assumptions, various combinations of parameter values may exist that yield the same quality of fit. Hence, there is a need for quantifying the uncertainty in estimated parameters and to ascertain the uniqueness of the found solution. This paper presents a stochastic method to estimate the parameters of an image-based electromechanical model of the heart and their uncertainty due to noise in measurements. First, Bayesian inference is applied to fully estimate the posterior probability density function (PDF) of the model. To that end, Markov Chain Monte Carlo sampling is used, which is made computationally tractable by employing a fast surrogate model based on Polynomial Chaos Expansion, instead of the true forward model. Then, we use the mean-shift algorithm to automatically find the modes of the PDF and select the most likely one while being robust to noise. The approach is used to estimate global active stress and passive stiffness from invasive pressure and image-based volume quantification. Experiments on eight patients showed that not only our approach yielded goodness of fits equivalent to a well-established deterministic method, but we could also demonstrate the non-uniqueness of the problem and report uncertainty estimates, crucial information for subsequent clinical assessments of the personalized models.
\end{abstract}

\section{Introduction}

Cardiomyopathy is one of the most common types of cardiovascular disease with significant mortality and morbidity rates [1]. However, clinical management of these patients is challenged by the wide variety of disease causes and therapies. Computational models of heart function are being explored to improve patient stratification, risk prediction and therapy planning [2] Yet, the high model complexity and the limited availability and often noisy measurements still hinder the necessary personalization of these models from being clinically useful.

P. Golland et al. (Eds.): MICCAI 2014, Part II, LNCS 8674, pp. 9-16, 2014.

(C) Springer International Publishing Switzerland 2014 
Intense research is being carried out to solve the inverse problem of cardiac modeling, i.e. the estimation of model parameters from clinical data for patientspecific computations. Solutions based on nonlinear least squares (NLS) minimization of a specialized cost function [3] are very popular, among other reasons due to the high availability of easy-to-use general purpose frameworks. On the other hand, more sophisticated methods to better deal with the high complexity of the problem are emerging [4.5.

Uncertainty in data and model assumptions are known to increase the nonidentifiability of parameters. In particular, solution uniqueness is not guaranteed and multiple solutions or entire manifolds of solutions with equal level of confidence may exist. As a result, the clinical value of one single estimate can be questioned. Yet, to the best of our knowledge, only little work exists in the cardiac modeling community which addresses these challenges. Parameter uncertainty quantification (UQ) due to noise in the data has been investigated in 677. for the cardiac electrophysiology problem. However, the authors do not take into account this knowledge to estimate a robust optimum for the model parameters under consideration as their main focus was on UQ. In [7, the mean of the posterior density was selected as parameter value, which can become inaccurate for skewed or multimodal distributions. In [6], the maximum posterior was used and evaluated on synthetic data only. It is not clear though whether that choice would be robust under uncertain data noise level.

This paper presents a stochastic method for the robust estimation of biomechanical parameters of the myocardium and their uncertainty due to noisy data. First, we estimate a surrogate model of an image-based electromechanical model of the heart by using Polynomial Chaos Expansion, which is then used in a Bayesian inference framework to estimate posterior probabilities of model parameters. Then, we apply mean-shift on the posteriors to find the optimal parameter value by integrating the space of measurement uncertainties. Experiments on eight dilated cardiomyopathy patients showed that our approach yielded goodness of fit equivalent to a well-established deterministic method while being as computationally efficient and providing confidence intervals. More importantly, we demonstrate that the manifold of possible solutions is much larger including multimodal posteriors, which are automatically identified, therefore enabling quantitative assessment of the clinical utility of estimated parameters.

\section{Method}

\subsection{Cardiac Electromechanical Model}

The model proposed in [8] is employed in this work. Cardiac geometry at end-diastasis is efficiently segmented under expert guidance from volumetric magnetic resonance images (Fig. 1). Endocardium and epicardium surfaces are detected using machine learning algorithms [9. A rule-based myocardial fiber architecture model is created, with fibers varying between $-80^{\circ}$ and $80^{\circ}$ from the epicardium to the endocardium. Cardiac electrophysiology (EP) is calculated using LBM-EP, a fast model based on the Lattice-Boltzmann method. 


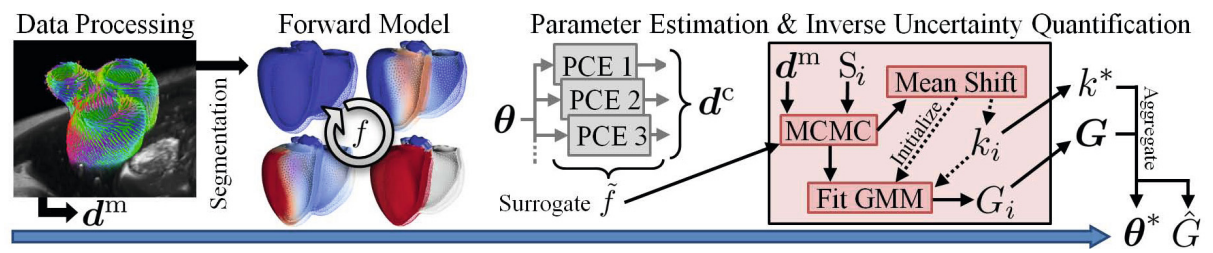

Fig. 1. Proposed robust estimation framework. From imaging and clinical data, the electromechanical forward model is personalized using an efficient surrogate model and inverse UQ-based parameter estimation. See text for details.

The Mitchell-Schaeffer cellular model is employed as a tradeoff between model fidelity and complexity. Cardiac hemodynamics (HD) is calculated using a lumped model of the intra-ventricular pressure varying according to models of arterial compliance (3-element Windkessel (WK) model) and atrial contraction. Cardiac biomechanics is computed using the finite element method to solve the dynamics equation $\mathrm{M} \ddot{\boldsymbol{u}}+\mathrm{C} \dot{\boldsymbol{u}}+\mathrm{K} \boldsymbol{u}=\boldsymbol{f}_{a}+\boldsymbol{f}_{p}+\boldsymbol{f}_{b} \cdot \ddot{\boldsymbol{u}}, \dot{\boldsymbol{u}}$ and $\boldsymbol{u}$ denote nodal accelerations, velocities and displacements, respectively. M, K and $\mathrm{C}$ are the mass, stiffness and Rayleigh damping matrices. Pressure force $\boldsymbol{f}_{p}$ is calculated from the HD model. The active stress $\boldsymbol{f}_{a}$ generated by the EP activated myocytes is computed using a phenomenological model of myocyte contraction [10, which is mainly governed by the maximum active stress parameter $\sigma_{0}$. Boundary conditions $\boldsymbol{f}_{b}$ capture heart attachment at the valve plane through springs. The orthotropic Holzapfel-Ogden (HO) constitutive law is used. The stress-strain energy is written as $\Xi=\beta \Psi\left(I_{1}, I_{4 f}, I_{4 s}, I_{8 f s}\right)$ where $\beta$ is a dimensionless factor to isotropically scale tissue stiffness, $I_{1}, I_{4\{f, s\}}$ and $I_{8 f s}$ are invariants of the deformation tensor (see [8] and references herein for HO parameter values).

The focus of this work is on biomechanics. Hence, patient's geometry, EP and HD parameters are assumed to be known for each patient. The geometry is obtained from the images. The WK parameters are estimated using the simplex method based on available invasive pressure measurements and flow derived from MRI-calculated volume variations. The EP model is personalized using a gradient-free NLS optimizer based on 12-lead ECG data [11. It should be stressed that the proposed approach is not limited to a particular model.

\subsection{Inverse Uncertainty Quantification}

The above model is reformulated as a statistical problem $f(\boldsymbol{\theta})=\boldsymbol{d}^{\mathrm{c}}$ where $\boldsymbol{\theta}$ and $\boldsymbol{d}^{\mathrm{c}}$ are random input (model parameters) and output (model responses) variables. Bayesian Calibration is used to infer the values of $\boldsymbol{\theta}$ and to quantify their uncertainty due to noisy measurements $\boldsymbol{d}^{\mathrm{m}}$. The goal is to compute the posterior $p\left(\boldsymbol{\theta} \mid \boldsymbol{d}^{\mathrm{m}}\right)$ by forward propagation of uncertainty [12]. Following Bayes' rule,

$$
p\left(\boldsymbol{\theta} \mid \boldsymbol{d}^{\mathrm{m}}\right) \propto p\left(\boldsymbol{d}^{\mathrm{m}} \mid \boldsymbol{\theta}\right) p(\boldsymbol{\theta})=\exp \left(-\frac{1}{2} \varepsilon\left(\boldsymbol{d}^{\mathrm{c}}, \boldsymbol{d}^{\mathrm{m}}\right)^{\top} \mathrm{S}^{-1} \varepsilon\left(\boldsymbol{d}^{\mathrm{c}}, \boldsymbol{d}^{\mathrm{m}}\right)\right) p(\boldsymbol{\theta}) .
$$


$p\left(\boldsymbol{d}^{\mathrm{m}} \mid \boldsymbol{\theta}\right)$ is the likelihood. It describes how well each set of parameters is supported by the data. $p(\boldsymbol{\theta})$ is the prior. It represents the knowledge on the parameters independently from the measurements, and is modeled in this work using a uniform distribution. As in [6], we estimate the likelihood $p\left(\boldsymbol{d}^{\mathrm{m}} \mid \boldsymbol{\theta}\right)$ by expressing it in terms of the error between responses and measurements, $\varepsilon\left(\boldsymbol{d}^{\mathrm{c}}, \boldsymbol{d}^{\mathrm{m}}\right)=\boldsymbol{d}^{\mathrm{c}}-$ $\boldsymbol{d}^{\mathrm{m}}$, modeled as a normal distribution $\mathcal{N}(\mathbf{0}, \mathrm{S})$. In this work, all sources of error are aggregated under $\varepsilon$. The posterior $p\left(\boldsymbol{\theta} \mid \boldsymbol{d}^{\mathrm{m}}\right)$ is then sampled using the Markov Chain Monte Carlo (MCMC) delayed rejection adaptive Metropolis [13] method.

To make the MCMC sampling computationally tractable, we use Polynomial Chaos Expansion (PCE) to estimate a surrogate model $\tilde{f}(\boldsymbol{\theta}) \approx f(\boldsymbol{\theta})$. An analogy of PCE is Fourier transform. Through multidimensional orthogonal polynomial approximations of $f, \tilde{f}$ provides an efficient functional mapping from model input to individual responses [14. For each response $r \in \boldsymbol{d}^{\mathrm{c}}$, the finite PCE approximation $\tilde{f}_{r}(\boldsymbol{\theta})$ corresponds to expressing that model response $f_{r}(\boldsymbol{\theta})$ in terms of linear combinations of polynomials, $f_{r}(\boldsymbol{\theta}) \approx \tilde{f}_{r}(\boldsymbol{\theta})=\sum_{p=0}^{P} \alpha_{p} \Psi_{p}(\boldsymbol{\theta})$ where the total number of coefficients $P+1=(q+1)^{\operatorname{dim} \theta}$ is defined by a user-specified maximum polynomial order $q$ (the higher, the more fidel the approximation). The coefficients $\alpha_{p}$ are obtained using spectral projection, which requires $P+1$ forward model runs. The multivariate polynomial basis $\Psi_{p}(\boldsymbol{\theta})$ is defined as combination of univariate $1 \mathrm{D}$ basis Legendre polynomials. The reader is referred to 1214 for theoretical details. The PCE surrogate is used to efficiently generate MCMC samples of $p\left(\boldsymbol{\theta} \mid \boldsymbol{d}^{\mathrm{m}}\right)$, instead of the full forward model.

\subsection{Posterior Analysis under Uncertainty}

From the surrogate model and the Bayesian calibration described above, we now have a PDF of the model parameters knowing the noise in the data, $p\left(\boldsymbol{\theta} \mid \boldsymbol{d}^{\mathrm{m}}\right)$. Coming back to our original objective, i.e. model personalization, we now need to derive, from that PDF, an estimate of the model parameters $\boldsymbol{\theta}^{*}$ and their confidence interval (Fig. 1). Additionally, we want to estimate the parameters that are most robust to varying level of noise in the data, since that level is often difficult to precisely estimate. To that end, we propose to aggregate the posteriors $p\left(\boldsymbol{\theta} \mid \boldsymbol{d}^{\mathrm{m}}\right)$ calculated for different level of noise into one PDF, from which we will estimate the most likely value of the model parameters.

More precisely, first we estimate the number of modes $k_{i}$ in the posterior $p\left(\boldsymbol{\theta} \mid \boldsymbol{d}^{\mathrm{m}}\right)$ using the mean-shift algorithm for a given level of noise $\mathrm{S}$ (Eq. 1), on which we fit a Gaussian mixture model (GMM) $G_{i}$ with $k_{i}$ components. This step is repeated $n_{\mathrm{S}}$ times with distinct levels of measurement noise uncertainty by varying the error variances of the individual responses in $\mathrm{S}=\mathrm{S}_{i}$ of the likelihood. At the end of this process, $n_{\mathrm{S}}$ mixture models $\boldsymbol{G}=G_{1} \ldots G_{n_{\mathrm{S}}}$ are generated.

In a second step, the $G_{i}$ are aggregated to get the final estimate. Consider one particular mixture model $G_{i} \in \boldsymbol{G}$. For each of the $j=1 \ldots k_{i}$ means $\boldsymbol{\mu}_{i j}$ of the components of $G_{i}$, we compute its support $\omega_{i j}=\sum_{t \neq i} \log G_{t}\left(\boldsymbol{\mu}_{i j}\right)$ by summation of the log-probabilities of $\boldsymbol{\mu}_{i j}$ in each of the other $G_{t}$. We normalize the $\omega_{i j}$ such that all values are mapped between 0 and 1 , the latter representing 
the highest support. Next, the $\boldsymbol{\mu}_{i j}$ are separated into $k^{*}$ clusters $\boldsymbol{K}=K_{1} \ldots K_{k^{*}}$ using $\omega_{i j}$-weighted $k$-means clustering. $k^{*}$ is determined by voting among all $k_{i}$ in $\boldsymbol{G}$. Finally, the centroid $\hat{\boldsymbol{\mu}}_{l}$ of the $\boldsymbol{\mu}_{i j}$ in the cluster with the highest combined support $\hat{\omega}_{l}=\sum_{i j \in K_{l}} \omega_{i j}$ is selected as the final estimate $\boldsymbol{\theta}^{*}=\hat{\boldsymbol{\mu}}_{l}$. This way, we increase the robustness in the estimate even without explicitly knowing the level of noise in the measurements, and are still able to capture multi-modal PDFs.

Uncertainty in estimated parameters is described by confidence regions. Let $\Upsilon_{i j}$ be the covariance matrix of the $j$ th mixture component in the $i$ th mixture model $G_{i}$. We assume that in each cluster $K_{l}$, all contained $\boldsymbol{\mu}_{i j}$ with corresponding $\Upsilon_{i j}$ are distorted (through noise) manifestations of the same normal PDF, which is centered at the centroid $\hat{\boldsymbol{\mu}}_{l}$ with unknown covariance $\hat{\Upsilon}_{l}^{*}$. We approximate $\hat{\Upsilon}_{l}^{*}$ by linear combination of the covariance matrices $\hat{\Upsilon}_{l}=\hat{\omega}_{l}^{-1} \sum_{i j \in K_{l}} \omega_{i j} \Upsilon_{i j}$, which is more robust to noise compared to using a single $\Upsilon_{i j}$. Finally, all information from the $k^{*}$ clusters is merged into one robust GMM $\hat{G}=\sum_{l=1}^{k^{*}} \hat{\omega}_{l} \mathcal{N}\left(\hat{\boldsymbol{\mu}}_{l}, \hat{\Upsilon}_{l}\right)$, thus forming an explicit representation of uncertainty.

\section{Experiments and Results}

Experimental Protocol. Our method was evaluated on eight dilated cardiomyopathy patients showing large variety of disease severity (left ventricular (LV) ejection fraction (EF) from 19\% to 47\%). Invasive catheterization was performed to measure LV pressure. Short-axis cine MRI stacks were used to estimate model anatomy and LV volume variation over time [9. The main objective was to investigate uniqueness and confidence levels of biomechanical parameter estimates given the measured data affected by unknown noise.

Cardiac electrophysiology and arterial parameters were estimated from 12 lead ECG and the pressure/volume data (Sec. 2.1). The proposed approach was then applied to estimate LV maximum active force and passive biomechanical tissue properties $\boldsymbol{\theta}=\left(\sigma_{0}, \beta\right)$. The responses chosen in that study were the minima, maxima and means of pressure/volume curves. PCE surrogates for the patientspecific electromechanical models were computed with maximum order $q=4$. Therefore, 25 true model evaluations on a $5 \times 5$ isotropic grid were performed, where $\sigma_{0}$ and $\beta$ varied within physiological ranges: $[150,330] \mathrm{kPa}$ and $[0.2,1.5]$.

The mean-shift based posterior analysis was carried out using $n_{\mathrm{S}}=15$ random noise levels. Intermediate GMMs (Sec. 2.3) were estimated based on $5 \cdot 10^{4}$ MCMC samples each. The response-specific level of noise was modeled as the standard deviation $S D$ of the assumed measurement error, individually drawn from a uniform distribution $S D_{r} \sim \mathcal{U}\left(r^{l}, r^{u}\right)$. Lower and upper bounds $r^{l}=$ 1 units and $r^{u}=3$ units, units denoting $\mathrm{mL}$ or $\mathrm{kPa}$ for volume and pressure responses, respectively, were chosen heuristically to model plausible levels of data noise. The error covariance matrix $\mathrm{S}$ in Eq. 1 is defined as $\operatorname{diag}\left(S D_{r_{1}}^{2}, S D_{r_{2}}^{2}, \ldots\right)$.

Precision of PCE-Based Surrogate Model. First, we verified that the selected PCE order $q=4$ was enough to model the posterior of the forward model. We compared the responses computed with the PCE model of order $4, \tilde{f}$, with 

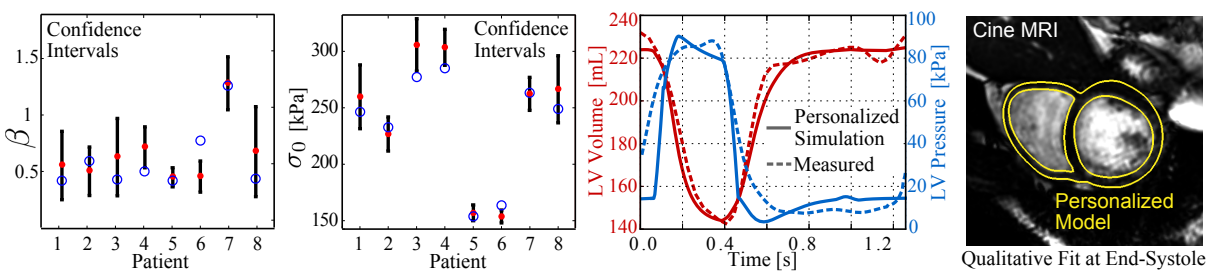

Fig. 2. Left: 95\% confidence intervals of GMM mode with highest support estimated by our approach. Red dots/blue circles represent our estimate/NLS solution, all of them within or near the confidence interval. Right: Plot showing measured versus simulated (using personalized model) LV volume/pressure curves, and qualitative comparison.

those obtained using a high-fidelity PCE model, $\tilde{f}^{\star}$, of order $q=10$ for all cases. 1000 parameters were sampled randomly per patient and all model responses were calculated using both $\tilde{f}$ and $\tilde{f}^{\star}$. Averaged over all patients, the errors over volume and pressure responses were $1.61 \mathrm{~mL}$ and $0.56 \mathrm{kPa}$ respectively, two orders of magnitude less than their absolute values. Furthermore, posterior PDFs estimated from the two surrogates compared qualitatively well (non-reported). These results suggested that $\tilde{f}$ was enough to reliably estimate model posterior.

Estimation of Biomechanical Parameters. We then estimated $\sigma_{0}$ and $\beta$ for all cases using our approach and verified the goodness of fit by comparing EF and stroke volume (SV) computed by the true model $f$ with the measurements. The estimated parameters yielded strong goodness of fit, with a mean EF error of $2.3 \% \pm 1.3 \%$ and $\mathrm{SV}$ error of $8.6 \mathrm{~mL} \pm 3.6 \mathrm{~mL}$. Fig. 2 reports the estimated parameters and $95 \%$ confidence intervals. As one can see, the noise in the data can have significant impact on the confidence of the estimated values, with in some cases intervals of up to $\pm 25 \mathrm{kPa}\left(\sigma_{0}\right)$ and $0.5(\beta)$. Such estimates could be employed as indicators of model fitting quality to suggest the operator to acquire more/better data to reduce them. Furthermore, our method is robust to varying noise levels (Fig. 3) and multimodal PDFs are captured correctly. The difference in EF and SV based on the two modes (Fig. 3) is only $0.2 \%$ and $1.6 \mathrm{~mL}$.

State-of-the-Art Parameter Estimation. Next, we compared the personalization results of the proposed estimator to those obtained using BOBYQA [15], a recently developed gradient-free optimizer. The employed objective function writes $\varphi\left(\boldsymbol{d}^{\mathrm{c}}\right)=\left\|\boldsymbol{d}^{\mathrm{c}}-\boldsymbol{d}^{\mathrm{m}}\right\|_{2}^{2}$. The NLS-based EF error is similar to our approach with $2.0 \% \pm 0.9 \%$, and the SV error is measured as $7.6 \mathrm{~mL} \pm 2.6 \mathrm{~mL}$ on the same datasets. On average, BOBYQA took $13.3 \pm 1.9$ iterations to converge, each involving a forward model run. Our approach requires 25 forward runs and is therefore computationally equivalent while providing uncertainty estimates.

Evaluation of Estimated Uncertainty. Finally, we conducted a synthetic experiment to verify the uncertainty estimates. Ground truth responses were generated using the forward model with fixed input parameters. Noise was added to the responses and the parameters were estimated from noisy responses using 



Fig. 3. Left: Example posterior for one dataset ( $80 \%, 95 \%$ and $99 \%$ confidence regions) estimated by our approach. The PDF is multimodal, i.e. the solution is not unique. Middle: $95 \%$ confidence intervals (for $\sigma_{0}$ ) of one patient with increasing measurement noise level $S D_{r}$. Although confidence intervals get larger (higher uncertainty), our estimate (red dot) remains robust. Right: Estimated confidence regions of our approach based on ground truth data (responses computed using forward model of one patient, $\left.\left(\sigma_{0}, \beta\right)=(240 \mathrm{kPa}, 0.85)\right)$, and various NLS solutions (dots) computed from the ground truth responses with added noise (noise drawn from $\mathcal{N}\left(0,3^{2}\right) \mathrm{kPa}$ or $\mathrm{mL}$ for each response). All but one (very high noise) estimates lie within regions of high confidence.

BOBYQA. Fig. 3] shows that all but one solutions lie within the estimated confidence regions. This promising result is a first step towards a comprehensive validation of the uncertainty estimates, which will be done in the future.

\section{Conclusion and Future Work}

In this paper, we explored the impact of data noise on the estimated biomechanical parameters. Our framework relies on stochastic parameter estimation and aggregates the probabilities estimated under different noise levels to derive a robust parameter estimate without explicitly knowing the level of noise in the data. While PCE requires the responses to possess finite second order moments, be smooth and with finite variance, the smooth physical nature of the model under consideration motivated our choice. Further theoretical analysis will be carried out to confirm the good behavior of the surrogate models. Yet, results on eight cases showed that not only our approach is as effective as well-established deterministic inverse method algorithms, but that it is also as computationally efficient while providing confidence intervals. Furthermore, we could demonstrate the non-uniqueness of the inverse problem by reporting different solution spaces, which can be automatically identified through the estimated posterior PDFs. Such an approach could therefore provide precious insights when analyzing the clinical relevance of estimated parameters and personalized model predictions. In addition to that, it could constitute a criterion to select and refine data acquisition protocols used for model personalization. Future work includes the independent modeling of data and model noise, and simultaneous estimation of more parameters, such as regional tissue properties or parameters from different model components, for instance by including Windkessel and cardiac electrophysiology parameters. 


\section{References}

1. McMurray, J., Adamopoulos, S., Anker, S., Auricchio, A., Dickstein, K., Falk, V., Filippatos, G., Fonseca, C., Gomez-Sanchez, M.: ESC guidelines for the diagnosis and treatment of acute and chronic heart failure. Eur. Heart J. 33(14), 1787-1847 (2012)

2. Trayanova, N.A.: Whole-heart modeling applications to cardiac electrophysiology and electromechanics. Circ. Res. 108(1), 113-128 (2011)

3. Prakosa, A., Sermesant, M., Allain, P., Villain, N., Rinaldi, C., Rhode, K., Razavi, R., Delingette, H., Ayache, N.: Cardiac electrophysiological activation pattern estimation from images using a patient-specific database of synthetic image sequences. IEEE TBME 61(2), 235-245 (2014)

4. Chabiniok, R., Moireau, P., Lesault, P.F., Rahmouni, A., Deux, J.F., Chapelle, D.: Estimation of tissue contractility from cardiac cine-MRI using a biomechanical heart model. Biomech. Model. Mechan. 11(5), 609-630 (2012)

5. Xi, J., Lamata, P., Lee, J., Moireau, P., Chapelle, D., Smith, N.: Myocardial transversely isotropic material parameter estimation from in-silico measurements based on a reduced-order unscented Kalman filter. J. Mech. Behav. Biomed. 4(7), 1090$1102(2011)$

6. Wallman, M., Smith, N.P., Rodriguez, B.: Computational methods to reduce uncertainty in the estimation of cardiac conduction properties from electroanatomical recordings. Med. Image Anal. 18(1), 228-240 (2014)

7. Konukoglu, E., Relan, J., Cilingir, U., Menze, B.H., Chinchapatnam, P., Jadidi, A., Cochet, H., Hocini, M., Delingette, H., Jaïs, P., Haïssaguerre, M., Ayache, N., Sermesant, M.: Efficient probabilistic model personalization integrating uncertainty on data and parameters: Application to eikonal-diffusion models in cardiac electrophysiology. Prog. Biophys. Mol. Bio. 107(1), 134-146 (2011)

8. Zettinig, O., et al.: From medical images to fast computational models of heart electromechanics: An integrated framework towards clinical use. In: Ourselin, S., Rueckert, D., Smith, N. (eds.) FIMH 2013. LNCS, vol. 7945, pp. 249-258. Springer, Heidelberg (2013)

9. Zheng, Y., Barbu, A., Georgescu, B., Scheuering, M., Comaniciu, D.: Four-chamber heart modeling and automatic segmentation for 3-D cardiac CT volumes using marginal space learning and steerable features. IEEE TMI 27(11), 1668-1681 (2008)

10. Sermesant, M., Delingette, H., Ayache, N.: An electromechanical model of the heart for image analysis and simulation. IEEE TMI 25(5), 612-625 (2006)

11. Zettinig, O., Mansi, T., Neumann, D., Georgescu, B., Rapaka, S., Seegerer, P., Kayvanpour, E., Sedaghat-Hamedani, F., Amr, A., Haas, J., Steen, H., Katus, H., Meder, B., Navab, N., Kamen, A., Comaniciu, D.: Data-driven estimation of cardiac electrical diffusivity from 12-lead ECG signals. Med. Image Anal. (2014)

12. Marzouk, Y.M., Najm, H.N., Rahn, L.A.: Stochastic spectral methods for efficient bayesian solution of inverse problems. J. Comput. Phys. 224(2), 560-586 (2007)

13. Haario, H., Laine, M., Mira, A., Saksman, E.: DRAM: efficient adaptive MCMC. Stat. Comput. 16(4), 339-354 (2006)

14. Adams, B.M., Dalbey, K.R., Eldred, M.S., Gay, D.M., Swiler, L.P.: DAKOTA, a multilevel parallel object-oriented framework for design optimization, parameter estimation, uncertainty quantification, and sensitivity analysis: Version 5.4 user's manual. Tech. rep., Sandia National Laboratories (2013)

15. Powell, M.J.: The BOBYQA algorithm for bound constrained optimization without derivatives. Tech. rep., University of Cambridge (2009) 